\title{
Correction to: Neural Engineering
}

\author{
Bin He
}

\section{Correction to:}

B. He (ed.), Neural Engineering, https://doi.org/10.1007/978-3-030-43395-6

The book was previously published with Electronic Supplementary Material content that was uploaded in error. The content has now been removed 